\title{
Software review:
}

\section{The system requirements and process impact of event-based marketing in financial services}

Received: 15th March, 2002

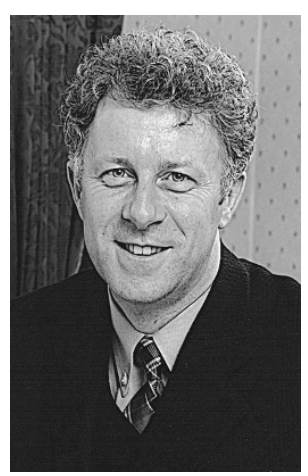

\section{Shaun Doyle}

is VP of Intelligent Marketing Solution at SAS. He is responsible for driving the application of SAS technologies in marketing. Prior to this role he was Chairman of Intrinsic, a campaign management software company. A co-founder of the company with Steve Treadwell, he was responsible for management of the business consultancy team and the strategic direction of the company. He has extensive experience in the design, development, implementation and exploitation of customer-focused database marketing systems in a number of business sectors. These include financial services, retail, leisure, charity and telecommunications. He has been involved in the design and building of over 50 marketing databases in blue chip organisations.

$\mathrm{He}$ is well known in the industry, regularly speaking and running specialist workshops at leading industry conferences. He has had a number of papers published in the UK and overseas on the application of database marketing.

Abstract This paper identifies the system requirements and marketing process impacts of trigger or event-based marketing in promoting products and services in a financial services organisation.

\section{INTRODUCTION}

A framework for classifying customer communications was described in a previous paper. ${ }^{1}$ This framework described five basic types of communication that need to be supported by a marketing database solution. These were:

- strategic communications

- tactical communications

- customer life cycle events

- product life cycle events

- customer trigger events.

In the case of strategic communications the marketing activities are part of an ongoing programme aimed at meeting a specific strategic objective such as:

- development of the brand

- growth of a new customer segment
- launch of a new product or service

- managing customer profitability

- migrating customers to the e-channel.

In the case of tactical communications the marketing activities are used to address a specific business issue, which is transient or short term in nature. Typical tactical communications are associated with:

- new branch opening or closure

- exploiting short-term competitive advantage

— spoiling competitive activities

- meeting business shortfalls

- changes in legal or government regulation

- changes in market environment.

The marketing activities in the case of customer life cycle events are focused around a customer or prospect life cycle 
event. Typical customer life cycle events are:

— birthdays

- changes in family status

- changes in employment status or type

- changes in wealth

— inheritance.

For product life cycle events the marketing activities are aimed at product life cycle events for current or historical customers. These events are normally associated with key dates or product-based transactions. Typical product life cycle events include:

- account opening

- account closure

- anniversary dates

- maturity dates

- renewal dates

- acquisition of a particular product combination.

In the case of customer trigger events the marketing activities are aimed at customer trigger events for current customers. These trigger events are generated as a result of an inward-bound communication from the customer (or third party, eg a solicitor) or a change in customer-bank behaviour. Typical customer trigger events would include:

- change of core customer data e.g. address changes

- customer complaint

- product or service information request, eg deed request, tax status change

- account activity, eg abnormal transaction.

This paper focuses on the last three types of communication. Collectively called trigger or event-based marketing their importance is growing as organisations recognise the value of making communications timely and relevant.

\section{DEFINITION}

The terms 'event' and 'trigger' have been used interchangeably. For the purpose of this paper the following definitions are used:

- a trigger is a change in data that could provide input into an event definition, eg change of address

- an event is defined by one or more triggers, eg change of address combined with change of marital status implying a customer life cycle event: marriage.

A trigger or an event may be used to drive a marketing communication where they provide evidence of a financial need that can be served by an organisation.

\section{IMPORTANCE OF CUSTOMERCENTRICITY}

Product life cycle events have played an important role in the past in driving marketing communications as they have been easy to execute, even when the organisation's system(s) are productcentric. Customer life cycle and customer trigger events on the other hand, have required the organisation to be able to execute customercentric communications. Generally speaking this is now becoming the norm, certainly in most financial service organisations and is facilitating customer life cycle and trigger event marketing activities.

\section{BENEFITS OF EVENT-BASED MARKETING COMMUNICATIONS}

If effectively executed these event-based marketing communication activities significantly out-perform traditional 
targeted communications covering a similar subject matter. The primary reason is that these communications are more likely to be timely and relevant. The trigger or event provides both time input and context input for the communication.

\section{EVENT-BASED MARKETING COMPONENTS}

The following section explores the key components required to support event-based marketing. These are:

- event analysis

- event detection

- event-based campaign management

- event opportunity measurement.

The following section looks as these components in more detail.

\section{Event analysis}

The identification of triggers and events was based on business rules with little or no statistical analysis going into identifying potential triggers or events. A number of organisations have, however, started using advanced statistical techniques to identify potential triggers on the marketing database. A range of statistical techniques have been used either to validate that a business rule-based trigger was predictive or to identify previously unknown triggers or groups of triggers (events).

The following statistical techniques should be supported:

— regression analysis

- tree analysis

- cluster analysis

- associative analysis

- neural networks

— pattern searching

- text mining.

Text mining has been used to search the content of e-mails being sent to a service centre to detect key words or combinations of words, which act as triggers for follow-up marketing communications.

A solution that is going to be used to support event-based marketing should be able to support the statistical validation of a business rule-based trigger and/or provide a statistically robust process for the identification of triggers.

The solution should include a set of business processes that structure the event analysis process for both business rule-based and statistically-derived triggers.

\section{Event detection}

Having identified that a particular trigger or set of triggers defines an event, the organisation has to put in place the necessary procedures to detect customers who have exhibited a particular trigger or group of triggers. This requires the application of rules against the customer base on a regular basis or in real time. The following types of data may be implicated:

- customer profile

- account

- account history

- customer account involvement

- account transactions

— operational contact history.

The detection process can be problematic. The following are some examples of the issues that may have to be addressed as part of any solution.

\section{Change history}

One of the difficulties with some trigger types is that they require the detection process to look for changes in a 
particular data item. This is difficult, as many marketing databases do not hold history of changes at a data item level, eg change in marital status. In such cases processes will need to be created that detect these changes or maintain a history on key data items.

\section{Query performance}

Looking for a particular pattern of transactions in a large marketing database, with $100 \mathrm{~m}$ transaction records will have implications for performance if not correctly architected. The solution must be designed with these performance requirements in mind. This may be achieved using 'raw horse power' and/or good database design.

\section{Volume of trigger rules}

Over time many businesses develop large libraries of trigger rules that need to be applied to the database. This will have implications for system performance and communication prioritisation. The solution should be able to apply a large number of rules on a daily basis and facilitate the maintenance of the trigger rules. The issue of prioritisation is covered later in the paper.

\section{Model scores}

Some organisations have found that changes in model scores provide valuable triggers for events. This means that large numbers of behavioural models (statistical procedures) may need to be run against the marketing database on a regular basis. This may have implications for system performance and the maintenance of model score history. The solution should support the use of models to drive trigger-based campaigns.

The solution should facilitate the estimation of trigger volumes so that a commercial assessment (business case) can be made before event-based campaigns can be established.

\section{Event-based campaign management}

Once a trigger or set of triggers has been identified the next step is to use these triggers to drive the execution of event-based campaigns. A single trigger could be used to drive a number of campaigns. The solution should support the following campaign management processes:

- maintenance of campaign reference data

- identification of target audience for campaigns

— prioritisation of campaigns

- application of global contact rules

- creation of communication cells within campaign

— definition of data requirements for communication

- execution of communication by relevant channel

- scheduling of campaign repetition

- campaign performance monitoring.

The development of a large number of event-based campaigns often results in the need to prioritise the communications that should be sent to a customer or prospect. This prioritisation process needs to take into account other communications that a customer is due to receive in the defined time window. If this is to be done the business needs to establish a consistent metric (eg target ROI or propensity to purchase) or set of business rules that the system can apply either at the campaign level or customer level.

It should be possible to integrate event-based campaigns with other campaign types within the same campaign management application. This will facilitate: 
- planning and management of all campaigns

- prioritisation across different campaign types

- consistency in measurement processes

- effective use of marketing and channel resources.

Event-based campaigns do drive some incremental requirements that the solution should support. These include the ability to:

- do test counts

- project future volumes for event-based campaigns (on a monthly, weekly or daily basis)

- prioritise within campaign type and then across campaign types

- apply global contact rules (these are rules that are used to frame the prioritisation process)

- execute small-volume campaigns (often requiring a higher degree of automation and tighter integration with channels)

- support more complex reporting requirements as the campaign may run for a longer period of time

- support more complex personalisation of the communication (the trigger provides an excellent context for the communication).

\section{Event opportunity measurement}

The use of global contact rules and campaign prioritisation results in a number of events not being progressed as opportunities. In order that the impact of these two processes can be measured and refined over time, there is normally a requirement to monitor the number of event-based opportunities that have not been progressed. The solution should:

— record all event-based opportunities detected
- flag those opportunities that have not been progressed and why

- allow the potential business value of the non-progressed opportunities to be determined

- simulate the impact of changes in: global contact rules; campaign priorities; customer communication priorities.

The solution should facilitate the recording of the lost opportunities and allow them to be progressed at a later stage where resource and business priorities allow.

\section{IMPACT OF EVENT-BASED MARKETING ON THE CAMPAIGN MANAGEMENT PROCESSES}

The adoption of event-based marketing affects both the technology and marketing business processes. The following section explores the impact that this type of approach has on the marketing processes.

The following is a typical process flow for marketing campaigns and the impact that event-based marketing has on the processes.

\section{Agree marketing plan}

The lack of clear understanding of the potential numbers of event-based communications that will be initiated in the coming year makes planning very difficult. This means that assumptions need to be made on likely volumes and response rates for a type of marketing activity that is primarily driven by the customer and not the business.

\section{Produce campaign plan}

The longitudinal nature of event-based marketing campaigns means care has to be taken to ensure that they do not 
conflict with other campaigns that are going to run in the year. Campaign priorities also need to be established at this stage to ensure that any likely conflicts can be taken into account during the planning stage. Some organisations also agree global contact rules at this stage in the process.

\section{Produce campaign business case}

An organisation's lack of ability to predict accurately the volumes and performance of event-based marketing campaigns means that they often make the business case using a range of values. These values are then used to set thresholds for campaign performance. If a campaign performance falls below these thresholds then the campaign will be terminated.

\section{Produce campaign brief}

The timely nature of event-based communications means that service level agreements (SLA) with both internal and external suppliers are often much tighter than for traditional campaigns. In order that these SLAs can be met, business processes will need to be refined.

\section{Manage response from supplier}

Separate contract frameworks are often used for the support of event-based marketing activities allowing the procurement process to be streamlined.

\section{Execute campaign}

The campaigns tend to be executed on a daily basis. This means that there is often a higher degree of automation and tighter integration with the communication delivery channels. The sign-off processes are also streamlined to allow things like copy and collateral to be revised in a more timely manner. Resource planning also has to be tightened to ensure sufficient resource is available to exploit the opportunities.

\section{Monitor campaign}

It is essential with event-based marketing campaigns that tighter monitoring processes are put in place to ensure that marketing can rapidly respond to changes in campaign performance. This is more critical because of the automated nature of the processes.

\section{Analyse campaign performance}

Post-campaign analysis often looks at additional marketing metrics surrounding lost opportunities, service levels and campaign performance. This is often complicated by the use of refined offers as the event-based campaigns are being executed.

\section{CONCLUSION}

Event-based marketing has an important role to play in driving customer communication in a modern financial services organisation. The timely and relevant nature of these communications means that performance is normally better than for traditionally targeted marketing communications. Delivering event-based marketing has an impact on the technology and processes supporting marketing and other functions. These need to be addressed if the final solution is to deliver business benefits.

(C) Shaun Doyle 2002

\section{Reference}

1 Doyle, S. (1998) 'A framework for classifying marketing communications and issues associated with supporting them in a marketing database solution', Journal of Database Marketing, Vol. 6, No. 1. 